Supporting information

\title{
Reconfigurable integrated optofluidic droplet laser array
}

Han Zhang, Prithviraj Palit, Yonghao Liu, Seyedmohsen Vaziri, and Yuze Sun*

Department of Electrical Engineering, University of Texas at Arlington

Arlington, TX 76019

*sun@uta.edu 


\section{Image processing for droplet size measurement}

As shown in Fig. S1, the original RGB image is directly captured by the camera. Through our home-developed MATLAB program, first, the RGB image is converted into a gray scale image. Second, gray scale image is normalized to the scale of $[1,255]$ (minimal value corresponds to 1 , maximal value corresponds to 255) to strengthen the contrast between droplet area and background area. Third, the normalized gray scale image is exported into ImageJ to perform the edge detection process. Fourth, after edge detection, a threshold is set to remove all the pixels that are below the threshold value in the background to further strengthen the contrast between droplet edge and background noise. Fifth, through a MATLAB built-in function "edge", the edge of the droplet is detected, and image is converted into a binary image. Sixth, inner area of the edge detected binary image is filled, and the logic value changes from "0" to " 1 ". Seventh, for a binary image the MATLAB built-in function "regionprops" is applied to find the droplet with center location and radius. Then the MATLAB-found circle and original RGB image are plotted together for comparison. The radius found in step 7 is used as the droplet size.

\section{Droplet size distribution of droplets with image-based method}




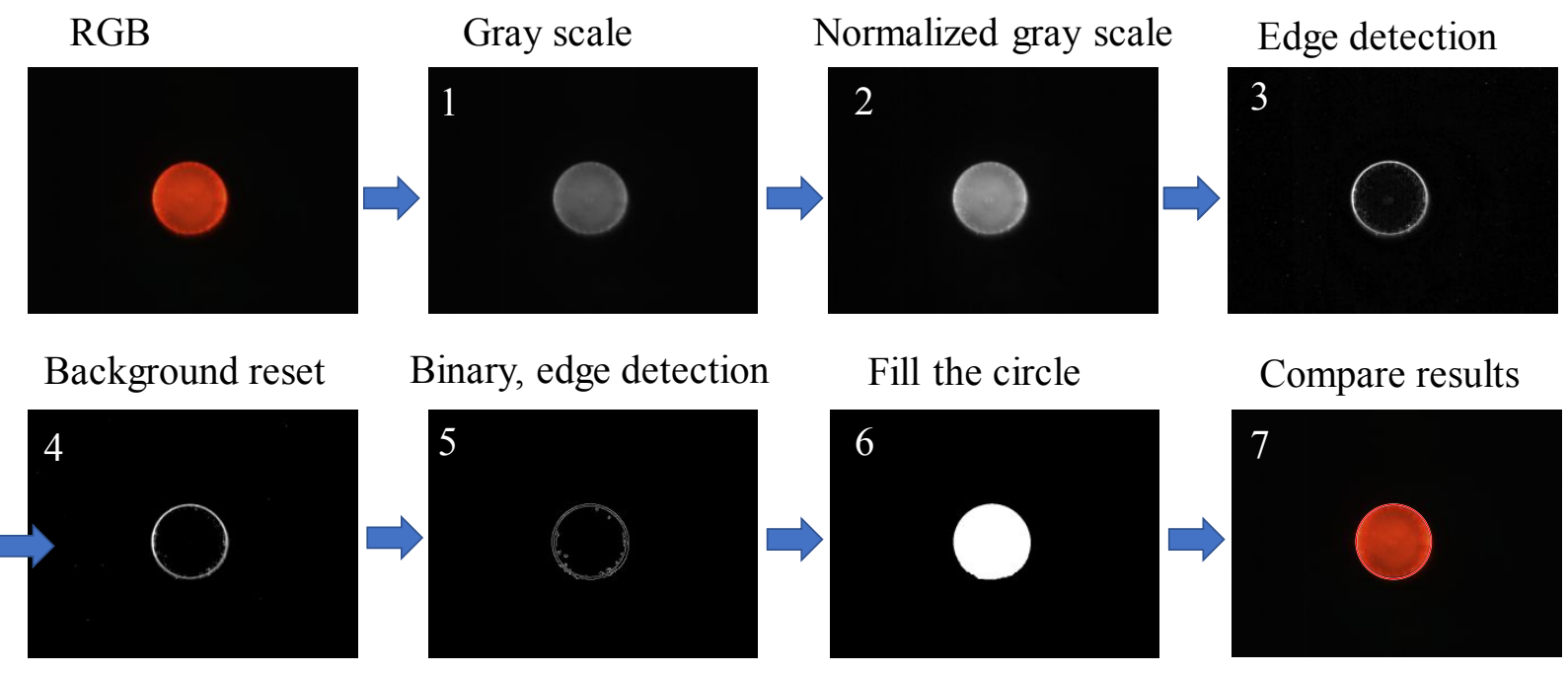

Figure S1 The process flow of droplet size measurement for our home-developed Matlab program. 


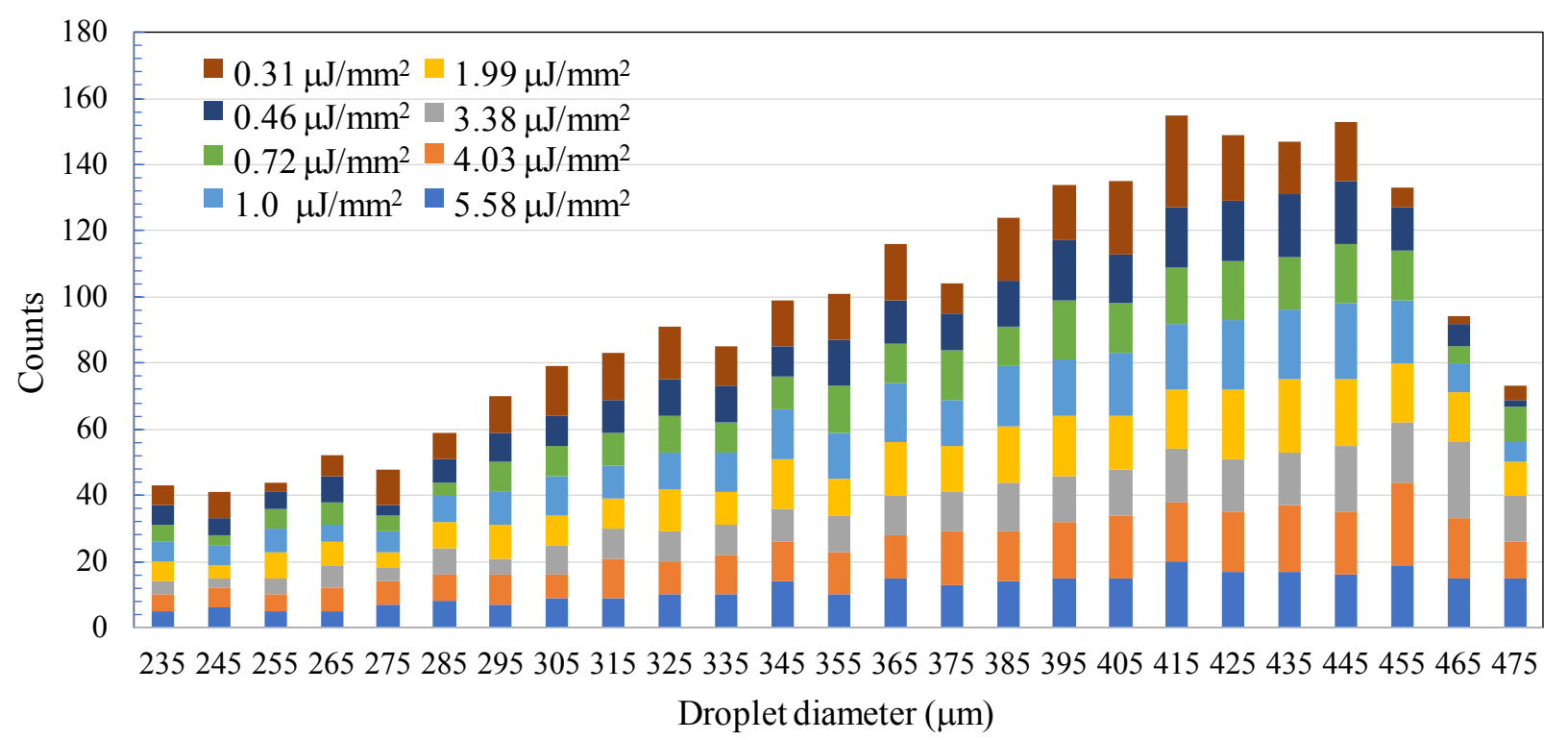

Figure S2 The size distribution of droplets under different excitation pump intensity. 